\title{
Effect of Poling Field and Non-linearity in Quantum Breathers in Ferroelectrics
}

\author{
Subhra Jyoti Mandal ${ }^{1}$, Vir Ranjan Kumar ${ }^{2}$, Arindam Biswas ${ }^{3}$, \\ A. K Bhandhopadhyay ${ }^{4}$, A K Bhattacharjee ${ }^{5}$, Durbadal Mandal ${ }^{5}$ \\ ${ }^{I}$ Dumkal Institute of Engineering and Technology, Dumkal, Murshidabad, Pin-742406 \\ ${ }^{2}$ Azmet College of Engineering and Technology, Kishanganj, Bihar- 855108, India \\ ${ }^{3}$ Dr. B. C Roy College of Engineering, Fuljhore, Durgapur, Burdwan \\ ${ }^{4}$ Bengal Institute of Technology and Management, Santiniketan Sriniketan, Bypass, P.O-Dwaranda, Birbhum, \\ West Bengal \\ ${ }^{5}$ National Institute of Technology, Durgapur, West Bengal, India, Pin-713209
}

\begin{abstract}
Lithium tantalate is technologically one of the most important ferroelectric materials with a low poling field that has several applications in the field of photonics and memory switching devices. In a Hamiltonian system, such as dipolar system, the polarization behavior of such ferroelectrics can be wellmodeled by Klein-Gordon $(K-G)$ equation. To probe the quantum states related to discrete breathers, the same $K-G$ lattice is quantized to give rise to quantum breathers (QBs) that are explained by a periodic boundary condition. The gap between the localized and delocalized phonon-band is a function of impurity content that is again related to the effect of pinning of domains due to antisite tantalum defects in the system, i.e. a point of easier switching within the limited amount of data on poling field.
\end{abstract}

Keywords: Lithium Tantalate, K-G Lattice, Quantum Breathers, Poling Field, Nonlinearity (Alpha)

\section{INTRODUCTION}

In the field of applied physics, one of the most investigated materials is ferroelectric, which has important applications as memory switching [1-4] and in nonlinear optical communications [5], non-volatile memory devices $[6,7]$, and many others $[\mathbf{8 , 9}$. Lithium tantalate with a low poling field appears to be a promising candidate as a key photonic material for a variety of devices: such as optical parametric oscillators, nonlinear frequency converters, electro-optics and second-order nonlinear optical material, holography, etc. Many of such applications include important nano-devices [9-11].

Ferroelectricity is an electrical phenomenon whereby certain materials exhibit a spontaneous dipole moment. The direction of this moment can be switched between the equivalent states by the application of an external electric field [1-4]. It arises in certain crystal systems that undergo second-order structural changes below the Curie temperature, which results in the development of spontaneous polarization. This can be explained by Landau-Ginzburg free energy functional $[\mathbf{3 , 4 , 9}$. The ferroelectric behavior is commonly explained by the rotation of domains and domain walls that are present in the crystal with uniform polarization [1-4]. This behavior is nonlinear in terms of hysteresis of polarization $(P)$ and electric field $(E)$ vectors.

Localization in a system is attributed either to its disorder or to its nonlinearity [13]. The first one, i.e. Anderson localization has been implemented in details in many types of device applications. As the nonlinearity arises in ferroelectrics in terms of $P-E$ hysteresis due to the rotational movement of the discrete domains and domain walls, they could also give rise to the localization. Hence, the above explanations are given to relate the localized waves of discrete breathers and domain walls in ferroelectric materials. The above description also shows the importance of domain wall in ferroelectrics in describing a soliton solution, i.e. nonlinear localized traveling waves that are robust and propagate without change in shape, giving the polarization profile and the distribution of the elastic strain across the domain wall [14]. On the other hand, discrete breathers (DBs) are discrete solutions, periodic in time and localized in space and whose frequencies extend outside the phonon spectrum [27--15, 28--16]. This is described by our discrete Hamiltonian [9, 12], as detailed later.

In this new approach, for the characterization of DBs or classical breathers $[\mathbf{1 7}, \mathbf{1 8}]$, the bulk system was the right tool, but when we are dealing with smaller systems, we have to use quantum physics, which brings us to the quantum breathers (QBs) [19]. Once generated, QBs modify system properties such as lattice thermodynamics and introduce the possibility of non-dispersive energy transport, as generally described for DBs [20]. These are observed in many systems viz. ladder array of Josephson junction for superconductors, BEC in optical lattices/nonlinear photonic lattices, interacting optical waveguides, cantilever vibrations in micromechanical arrays, DNA, split-ring resonator (SRR) based metamaterials in antenna arrays, two-magnon bound states in antiferromagnets, two-phonon bound states (TPBS), i.e. quantum breathers, in ferroelectrics (see Ref. [21]). 
A brief account is given here on phonon bound state or breather state. Despite our work on discrete breathers [9, 18], so far the pinning has been explained classically, thereby prompting us to think about quantum explanations that have been briefly explored for ferroelectrics, such as lithium niobate with the impurity data [21]. Although impurity data for lithium tantalate (as antisite tantalum defects) are not available, we could work through Landau coefficient or nonlinearity route to explore if there is also pinning in such systems (see later). For quantum breathers, it is important to consider detailed information on phonons and their bound state concept, which is sensitive to the degree of nonlinearity. In the eigenspectrum or more traditionally $E_{k}$ vs $k$ plot, a quantum breather band separates itself from the delocalized phonon-band. Or in other words, it is the hopping tendency of the phonons that describe the quantum breathers. So, let us consider that the phonons in one sublattice may hop from one domain to another adjacent domain. This hopping might have some consequences with the change of nonlinearity or poling field for 'switching' that is again related to the impurity in the lattice, thereby the 'hopping strength' can be directly related to this phenomenon. It is determined by finding the phonon-band energy gap (i.e. the energy gap between the delocalized and localized phonons) in the usual eigenspectrum $[15,22]$.

The paper is organized as follows: in the Section II, we present the general mathematical model for TPBS parameters and finally after second quantization on K-G lattice is done with Bosonic operators along with our method of computation. In Section III, the results and discussion are also presented. In Section IV, the conclusions are given.

\section{THEORETICAL DEVELOPMENT}

In a previous treatment, a time-dependent formulation for the dynamics of the domain array was obtained as a generalization of the Landau-Ginzburg free energy form involving polarization $(P)$ and electric field $(E)$ vectors [12]: The nearest neighbor domains [i.e. the polarization in the $i$ th domain $\left(P_{i}\right)$ with that in the $(i-1)$ th domain $\left.\left(P_{i-1}\right)\right]$ were taken to interact by a harmonic potential with a phenomenological spring constant $k$ so that the resulting Hamiltonian for the polarization is given by $[\mathbf{9 , 1 2 ]}$ :

$$
H=\sum_{i=1}^{N}\left(\frac{1}{2 m_{d}}\right) p_{i}^{2}+\sum_{i=1}^{N} \frac{k}{4}\left(P_{i}-P_{i-1}\right)^{2}+\sum_{i=1}^{N}\left(\left(-\frac{\alpha_{1}}{2} P_{i}^{2}+\frac{\alpha_{2}}{4} P_{i}^{4}\right)-E P_{i}\right)
$$

Eq. (1) gives a general treatment of the mode dynamics in the array, particularly for modes, which are strongly localized over a small number of domains in the array. For extended modes and modes which are localized, and slowly range over a large number of consecutive domains, our discrete Hamiltonian [Eq. (1)] can be split as:

$$
\widetilde{H}=H_{0}+H_{1}
$$

Where, $H_{0}=\sum_{i} \frac{p_{i}^{2}}{2}-\frac{\left(\alpha_{1}+\lambda\right) P_{i}^{2}}{2}+\frac{\alpha_{2} P_{i}^{4}}{4}-E P_{i}$

$H_{1}=-\frac{\lambda}{2} \sum_{i} P_{i} P_{i-1}$

Hence, for $n$-particles or $n$-levels in the anharmonic potential well, a general basis may be written as:

$\mid \vec{n}>=\psi_{n_{1}}\left(x_{1}\right) \psi_{n_{2}}\left(x_{2}\right) \ldots \psi_{n_{f}}\left(x_{f}\right)$

and $<\vec{m}|H| \vec{n}>=<\vec{m}\left|H_{0}\right| \vec{n}>+<\vec{m}\left|H_{1}\right| \vec{n}>$

For the present numerical analysis, the Fourier grid Hamiltonian method [22] with 1000 grids and 0.006 spacing to calculate various eigen values and eigenvectors at the grid points. For the present calculations, we restrict ourselves to two phonon states, since at the working temperature the number of phonon is very small. In order to reduce the computer memory requirement, we take the advantage of translational invariance by periodic Bloch

wave formulation $\left|\psi>=\sum_{j} v_{j}\right| \varphi_{2}^{j}>$, as detailed in Ref. [21, 22]. Due to translational invariance, the eigenstates of $H$ are also eigenstates of the translation operator $T$, where: $\tau=\exp (i q)$ is its eigenvalue with $q=\frac{2 \pi v}{f}$ being allowed Bloch wave number and $v \in\left[-\left(\frac{f-1}{2}\right),\left(\frac{f-1}{2}\right)\right]$. Here, $\tau$ is the eigenvalue of $T$, and $f$ is a renormalizing constant. Thus, we can construct the Bloch states: $\left|\varphi_{2}^{j}>=\frac{1}{\sqrt{f}} \sum_{s=1}^{f}\left(\frac{T}{\tau}\right)\right| 10 \ldots 01 \ldots .$. 
With this basis, we can derive the eigenenergies for each given Bloch wave number $q$ from: $H_{q}\left|\psi_{n}>=E\right| \psi_{n}>$. Hence, 3 could be written as:

$$
<\vec{m}|H| \vec{n}>=\sum \delta_{i, i} E_{i}-\frac{\lambda}{2} \sum_{i}\left[\exp (-i j q)\left(\prod_{i} \delta_{m_{i}, n_{i}}\right) D_{m_{i-1} n_{i-1}} D_{m_{i} n_{i}}\right]
$$

, where $q$ is the Bloch-wave number and $D_{m_{i} n_{i}}=\sum_{i} x_{i} \psi_{m_{i}}\left(x_{i}\right) \psi_{n_{i}}\left(x_{i}\right), \psi_{m_{i}}$ 's and $E_{i}$ 's are eigenvectors and eigenvalues obtained from Fourier grid Hamiltonian method. Here, due to symmetric and asymmetric nature of the eigenfunctions $D_{m n}=0$, if $(m-n)=0$. Hence, for a two-phonons case, the non-zero hopping coefficients are $D_{01}=D_{10}, D_{12}=D_{21}$. The energy gap between the single phonon continuum and a bound state is given by:

$$
E_{g}=E_{2}-E_{0}-2\left(E_{1}-E_{0}\right)
$$

where $E_{0}, E_{1}$ and $E_{2}$ are three eigenvalues at different points of wave vector $(k)$ that are calculated from our computation to generate $E(k)$ vs. $k$ curve, which gives the signature of quantum breathers $(\mathrm{QB})$ in terms of twophonon bound state. The width of the single-phonon in the eigenspectrum is given by the magnitude of $4 \sigma$ i.e the width of the single delocalized phonon-band, where $\sigma$ is expressed as:

$$
\sigma=-\frac{\lambda}{2} D_{01}^{2}
$$

where $\lambda$ is an interaction term (i.e. equivalent to $k$ ). $D_{01}$ represents the coefficient for zero to single phonon generation. The variation of the single phonon spectrum width $\left(W_{p h}\right)$ represents (through $\left.D_{01}=D_{10}\right)$ the creation of a new phonon or annihilation of an existing phonon.

\section{RESULTS AND DisCUSSION}

The eigenspectra showing TPBS in lithium tantalate are shown in Figs. 1 to 3 for a poling field value of $1.60,17$ and $210 \mathrm{kV} / \mathrm{cm}$ respectively. The coupling value is taken as constant at 10 for all the spectra. The Landau parameter $(\alpha)$ is inversely proportional to the impurity content or poling field. In the absence of the impurity data, we would operate through Landau coefficient (read, nonlinearity) which should also give a correct picture of QBs in terms of various TPBS parameters, as nonlinearity and discreteness give rise to quantum localization or rather to the formation of QBs.

It is clearly seen from Figs. 1 to 3 that as the nonlinearity decrease, i.e. the poling field increases, the shape of the single-phonon continuum also changes in terms of its width that could be considered as significant, as it shows a functional dependence on nonlinearity. This width is measured by $W_{p h}$ shows a slight decrease towards lower $\alpha$ values $(\alpha=514$ to 421 . i.e. average value $=467)$ and then it drops quite drastically towards lower $\alpha$ values, i.e. the highest poling field of $210 \mathrm{kV} / \mathrm{cm}$. This is in contrast to that in lithium niobate system, where it sharply drops towards $\alpha=471$ and then it sharply increases towards lower $\alpha$ values [34]. This may be due to the different levels of the degree of polarization in the respective systems [49]. It also indicates that in lithium niobate, as the nonlinearity decreases from 1767 towards 471 , the difference in values of $W_{p h}$ is about 19.2 in absolute term, whereas that for lithium tantalate system, it is only about 1 for nonlinearity values from 4427 to 467 (average).

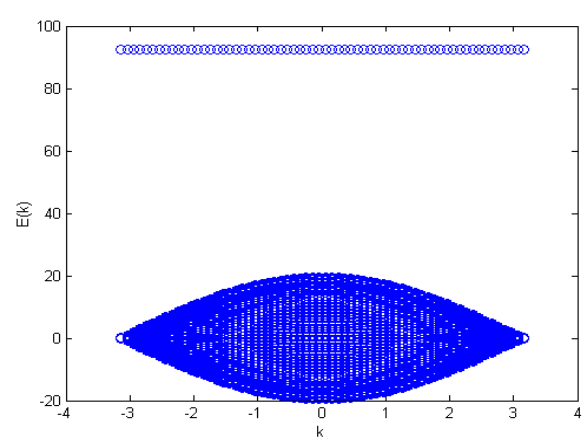

Fig 1: Eigenspectrum for a coupling value of $\bar{k}=10$, for a low poling field $E_{c}=1.6 \mathrm{kV} / \mathrm{cm}$ and $\bar{\alpha}=4427$. The continuum represents single phonon continua and the quantum breather band or two-phonon bound state is in the upper branch of the spectrum. 


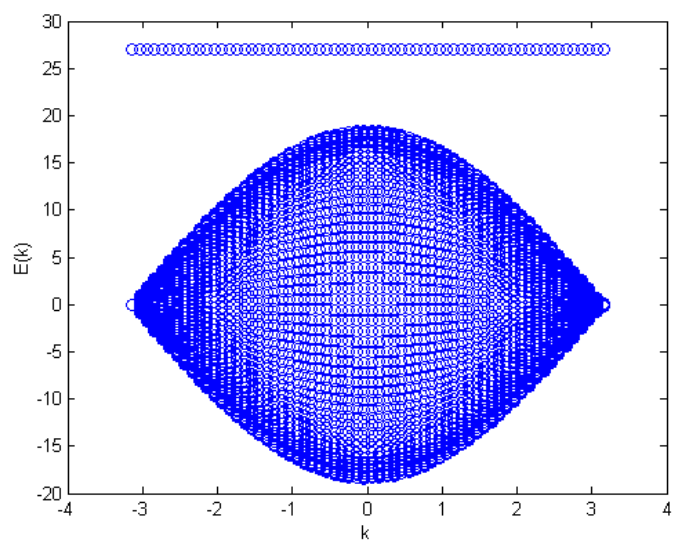

Fig. 2: Eigenspectrum for a coupling value of $\bar{k}=10$, for a moderate poling field $E_{c}=17 \mathrm{kV} / \mathrm{cm}$ and $\bar{\alpha}=421$. The similar spectra are observed with the effect of a decrease of nonlinearity in the single-phonon width.

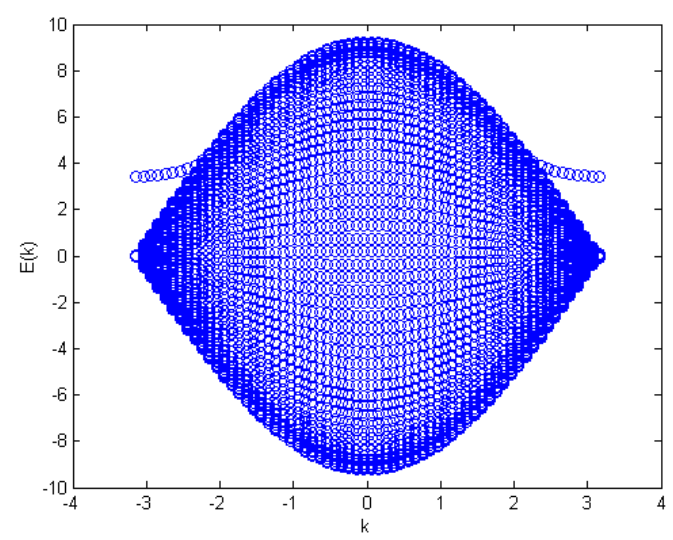

Fig. 3: Eigenspectrum for a coupling value of $\bar{k}=10$, for the highest poling field $E_{c}=210 \mathrm{kV} / \mathrm{cm}$ and lowest nonlinearity $\bar{\alpha}=37$. The similar spectra are observed with the effect of a further decrease of nonlinearity in the single-phonon width and in the appearance of a pseudo gap.

Table I

\begin{tabular}{|c|c|c|}
\hline (Poling Field in kV/cm) & $\alpha$ & $|\mathbf{E g}|$ \\
\hline 1.60 & 4426.68 & 92.45 \\
\hline 17.00 & 420.76 & 26.95 \\
\hline 210.00 & 37.09 & 3.44 \\
\hline
\end{tabular}

From the table 1 calculated by fig 1 to 3 , it is clearly shows that energy gap is increased by increasing poling field and decreasing nonlinearity i.e $\alpha$.

\section{CONCLUSION}

For quantum breathers in lithium tantalate, in a periodic boundary condition approach, the second quantization gives rise to some interesting values of TPBS parameters against nonlinearity within the system. This shows that after a value of nonlinearity (421), i.e. equivalent to a poling field of $17 \mathrm{kV} / \mathrm{cm}$, the formation of quantum breathers starts becoming relatively difficult thereby increasing single-phonon hopping energy that is observed upto this poling field and thereafter it increases monotonically toward higher values, whereas the energy gap after showing a small increase upto this poling field increases rapidly towards higher values; this was in contrast to that shown in lithium niobate system. This piece of information is considered useful for a future study in this new field of investigation of quantum breathers in ferroelectrics and other applications of QBs in important nonlinear optical materials. 


\section{REFERENCES}

[1] H. Fu, R.E. Cohen, Polarization rotation mechanism for ultrahigel ectromechanical response in single-crystal piezoelectrics, Nature , 403, 2000, 281-283.

[2] M.E. Lines, A.M. Glass, "Principles and Applications of Ferroelectrics and Related Materials" (Clarendon, Oxford, 1977).

[3] S. Kim, V. Gopalan, A. Gruverman, "Coercive fields in ferroelectrics: A case study in lithium niobate and lithium tantalite" Appl. Phys. Lett. ,80, 2002, 2740-2742.

[4] A.K. Bandyopadhyay, P.C. Ray, "Perturbation analysis and memory in ferroelectric materials", J. Appl. Phys., 95, 2004, $226-230$.

[5] Kevin T. Gahagan, Venkatraman Gopalan, Jeanne M. Robinson, Quanzi X. Jia Terence E. Mitchell, Matthew J. Kawas, Tuviah E. Schlesinger, and Daniel D. Stancil“"Integrated Electro-Optics Lens/Scanner in a $\mathrm{LiTaO}_{3}$ Single Crystal "Appl. Opt. 38, 1999, 11861190 .

[6] M. Dawber, K.M. Rabe, J. F. Scott, "Physics of thin-film ferroelectric oxides” Rev. Mod. Phys. 77, 20051083 -1130.

[7] G. Catalan, A. Schilling, J.F. Scott, J.M. Greg, "Domains in three-dimensional ferroelectric nanostructures: theory and experiments"J. Phys: Cond. Matter ,19, 2007, 132201-132207.

[8] S. Kim, V. Gopalan, K. Kitamura and Y. Furukawa, "Domain reversal and nonstoichiometry in lithium tantalate", J. Appl. Phys. ,90 ,2001, $2949-2963$.

[9] A. K. Bandyopadhyay, P. C. Ray, Loc Vu-Quoc and A. R. McGurn, "Multiple-time-scale Analysis of Nonlinear Modes in Ferroelectric LiNbO 3 ", Phys. Rev. B 81, 2010, 064104-064114.

[10] "Nanoelectronics and Information Technology", Ed. Rainer Waser (Wiley, Weinhiem, 2005).

[11] P. Giri, S. Ghosh, K. Choudhary, Md. Alam, A. K. Bandyopadhyay, P.C. Ray, "Importance of damping on nanoswitching in $\mathrm{LiNbO}_{3}$ type ferroelectrics "Phys. Scripta. ,83, 2011, 015702.

[12] A. K. Bandyopadhyay, P. C. Ray, and V. Gopalan, "An Approach to Klein-Gordon Equation for Dynamic Study in Ferroelectric Materials", J. Phys: Condens. Matter 18, 2006, 4093-4099.

[13] J. W. Fleischer, M. Segev, N. K. Elfremidis and D. N. Christodoulides, "Observation of 2-Dimensinal Discrete Solitons in Optically Induced Nonlinear Photonic Lattices", Nature 422,2003, 147-150.

[14] T. Dauxois, M. Peyrard, "Physics of Solitons", (Cambridge Univ. Press, Cambridge, 2006) pp211.

[15] S. Flach, A.V. Gorbach, "Discrete Breathers-Advances in Theory and Applications"Phys. Rep. 467, 2008, 1-116.

[16] M. Sato, B. E. Hubbard and A. J. Sievers, "Nonlinear Energy Localization and its Manipulation in Micromechanical Oscillator Arrays", Rev. Mod. Phys. 78, 2006, 137-157.

[17] A. Biswas, K. Choudhary, A.K. Bandyopadhyay, A.K. Bhattacharjee and D. Mandal, "Dissipative Breathers Modes in NanoFerroelectrics" J. Electron. Devices. 10. 2011, 423-428.

[18] P. Maniadis, G. Kopidakis and S. Aubry, "Classical and Quantum Targeted Energy Transfer between Nonlinear Oscillators", Physica D 188, 2004, 153-177.

[19] P. Giri, K. Choudhary, A. Sengupta, A. K. Bandyopadhyay and P. C. Ray, "DiscreteBreathers in Nonlinear LiNbO 3 -type Ferroelectrics", J. Appl. Phys. 109, 2011, 054105-0541012.

[20] A. C. Scott, J.C. Eilbeck and H. Gilhøj, "Quantum Lattice Solitons”, Physica D. 78, 1994, 194-213.

[21] R. A. Pinto, M. Haque and S. Flach, "Edge-Localized States in Quantum 1-Dimensional Lattices", Phys. Rev. A 79, 2009, 052118052125

[22] G. P. Tsironis, "If Discrete Breathers is the Answer, What is the Question?”, Chaos 13, 2003, 657-666.

[23] A. Biswas, K. Choudhary, A. K. Bandyopadhyay, A .K. Bhattacharjee and D. Mandal,"Quantum Pinning-transition due to Charge Defects in Ferroelectrics", J. Appl. Phys.110, 2011, 024104-024108.

[24] J. P. Nguenang, R. A. Pinto and S. Flach , "Quantum q-Breathers in a Finite Bose-Hubbard Chain: The Case of Interacting Bosons", Phys. Rev. B 75, 2007, 214303-214308. 